\title{
An ingredient for the elixir of youth
}

\author{
Cell Research (2014) 24:1381-1382. doi:10.1038/cr.2014.107; published online 12 August 2014
}

\begin{abstract}
Emerging evidence indicates that there are factors within the blood of young animals that have the ability to restore youthful characteristics to a number of organ systems in older animals. Growth/differentiation factor 11 (GDF11) is the first of such factors to be identified, and two new studies demonstrate that this "factor of youth" rejuvenates stem cells found in the skeletal muscle and brain of aged mice.
\end{abstract}

There may actually be some truth to the centuries-old myth that the blood of the young can restore youth. Early evidence came from a surgical procedure called heterochronic parabiosis, wherein the circulatory system of a young animal is coupled to that of an aged animal (Figure 1). This procedure can rejuvenate a number of organs in the older of the pair [1-3]. The provision of young blood even enhances cognitive performance in aged mice by improving synaptic plasticity in the hippocampus [4]. Conversely, aged blood has negative effects on the brain and skeletal muscle of young animals $[5,6]$. Thus, accumulating evidence has indicated that the blood of young animals contains powerful "factors of youth."

Recent work regarding age-related cardiac hypertrophy identified growth/ differentiation factor 11 (GDF11) as one such factor with rejuvenating powers. As animals become older, levels of circulating GDF11 normally decline. Remarkably, injecting GDF11 into aged mice recapitulates the effects of heterochronic parabiosis, reversing cardiac hypertrophy [7]. However, it remained unclear whether the effects of GDF11 were unique to the heart.
Sinha et al. [8] have now shown that increasing the systemic levels of GDF11 in aged mice also has rejuvenating effects on skeletal muscle. Aged mice injected daily with recombinant GDF11 (rGDF11) for four weeks have greater numbers of satellite cells, the local muscle stem cell population. Moreover, these satellite cells have less DNA damage and generate more myogenic cells in culture. rGDF11 supplementation also improves the in vivo regenerative capacity of satellite cells, resulting in the growth of larger muscle fibers after injury. Treatment with rGDF11 even increases exercise endurance and grip strength, demonstrating that the improvements seen in satellite cells relate to a functional enhancement in muscle performance. While it remains unclear whether these results are due primarily to effects on skeletal muscle, particularly given the known enhancement of cardiac function observed with rGDF11 treatment, this work demonstrates that a single systemic factor can help restore physiological properties of youth.

Studies regarding the rejuvenating capacity of young blood and rGDF11 have also been extended to the aged brain by Katsimpardi et al. [9]. The authors focused on the adult neural stem cells (NSCs) of the subventricular zone (SVZ) and found that heterochronic parabiosis enhances proliferation of Sox $2^{+}$NSCs in the aged mice. SVZ NSCs differentiate into neuroblasts that migrate to the olfactory bulb, and heterochronic parabiosis almost doubles the number of new neurons in the olfactory bulb of aged mice. Interestingly, these mice exhibit improved olfactory discrimination, but whether this behavioral change results directly from the enhanced neurogenesis or more generally to the whole-animal effects of heterochronic parabiosis is not yet known.

An additional change noted in the aged animals is an improved cerebrovascular architecture following heterochronic parabiosis, which appears to reverse the decline in blood vessel volume that normally occurs with aging. This increase in cerebral blood vessel volume is partially recapitulated with rGDF11 treatment. Cell culture experiments suggest that this effect is due to rGDF11-induced activation of the TGF- $\beta$ signaling pathway in brain capillary endothelial cells, increasing their proliferation. rGDF11 treatment also increases the number of Sox $2^{+}$ cells in aged SVZ, though not to the extent observed with heterochronic parabiosis. These results indicate that the beneficial effects of GDF11 are not limited to muscle, and will likely spur future work regarding its effect on other organ systems.

These studies offer compelling evidence that effects of aging can be reversed. However, it remains to be determined whether GDF11 acts directly on muscle satellite cells and NSCs in the brain, or whether the improvements in these stem cell populations are the indirect consequence of systemic effects. For instance, rGDF11 treatment improves cerebrovascular architecture and directly increases the proliferation of brain endothelial cells, which may indirectly enhance adult neurogenesis. Nevertheless, regardless of whether or not GDF11 can directly activate NSCs in the aged human brain, its effects on 


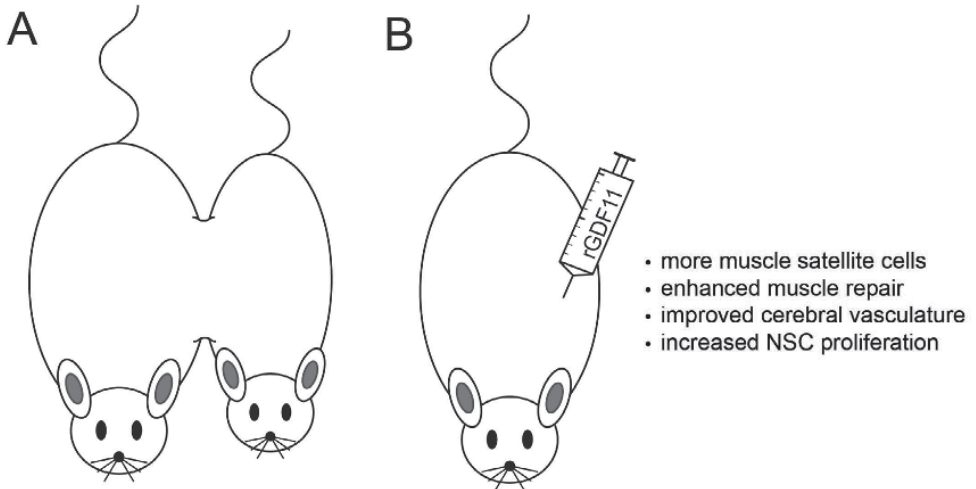

Figure 1 GDF11 rejuvenates aged skeletal muscle and brain. (A) Heterochronic parabiosis, which couples the circulatory systems of a young and old mouse, can restore youthful properties to many aged organs. (B) Treatment with rGDF11 alone revitalizes the skeletal muscle and brain of aged mice, resulting in functional improvements in strength and odorant detection.

the cerebral vasculature may potentially ameliorate microvascular ischemic brain disease, which has been linked to cognitive decline in the elderly [10].

It will also be important to address whether long-term systemic treatment with rGDF11 has any negative consequences, especially since GDF11 is known to regulate cell proliferation in the development of multiple organ systems. Perhaps there is a "reason" that this factor normally decreases with age. Additionally, while GDF11 can restore youthful characteristics to muscle, brain, and heart, this factor alone is generally less effective than heterochronic parabiosis. The increased efficacy of parabiosis may be due to the young blood providing additional beneficial factors, or to a dilution of detrimental components that accumulate with age. Therefore, identifying circulating factors that contribute directly to aging will potentially be of great importance in the search for therapies to restore youth.

As we move forward toward testing whether rGDF11 is indeed a powerful ingredient for the long sought-after elixir of youth, it will be important to determine whether the natural decline in circulating GDF11 serves any beneficial purpose. Establishing cell types that are directly affected by GDF11 may inform future work to design alterna- tive therapies that do not rely on the use of rGDF11. Regardless of whether GDF11 treatment proves to be an effective strategy of combatting aging in humans, these studies offer hope that the "inevitable" process of aging may actually be reversible.

\section{Rebecca E Andersen 1,2,3, Daniel A Lim ${ }^{1,2,4}$}

${ }^{1}$ Department of Neurological Surgery, ${ }^{2}$ Eli and Edythe Broad Center of Regeneration Medicine and Stem Cell Research, ${ }^{3}$ Developmental and Stem Cell Biology Graduate Program, University of California, San Francisco, San Francisco, CA 94143, USA; ${ }^{4}$ San Francisco Veterans Affairs Medical Center, San Francisco, CA 94121, USA Correspondence: Daniel A Lim

E-mail: LimD@neurosurg.ucsf.edu

\section{References}

1 Finerty JC. Physiol Rev 1952; 32:277-302.

2 Tauchi H, Hasegawa K. Mech Ageing Dev 1977; 6:333-339.

3 Conboy IM, Conboy MJ, Wagers AJ, et al. Nature 2005; 433:760-764.

4 Villeda SA, Plambeck KE, Middeldorp J, et al. Nat Med 2014; 20:659-663.

5 Villeda SA, Luo J, Mosher KI, et al. Nature 2011; 477:90-94.

6 Brack AS, Conboy MJ, Roy S, et al. Science 2007; 317:807-810.

7 Loffredo FS, Steinhauser ML, Jay SM, et al. Cell 2013; 153:828-839.

8 Sinha M, Jang YC, Oh J, et al. Science 2014; 344:649-652.

9 Katsimpardi L, Litterman NK, Schein PA, et al. Science 2014; 344:630-634.

10 Selnes OA, Vinters HV. Nat Clin Pract Neurol 2006; 2:538-547. 